LAW RENCE LIVERMORE N A TIO N A L LABORATORY

Oracle Log Buffer Queueing

A. S. Rivenes

February 7, 2005 
This document was prepared as an account of work sponsored by an agency of the United States Government. Neither the United States Government nor the University of California nor any of their employees, makes any warranty, express or implied, or assumes any legal liability or responsibility for the accuracy, completeness, or usefulness of any information, apparatus, product, or process disclosed, or represents that its use would not infringe privately owned rights. Reference herein to any specific commercial product, process, or service by trade name, trademark, manufacturer, or otherwise, does not necessarily constitute or imply its endorsement, recommendation, or favoring by the United States Government or the University of California. The views and opinions of authors expressed herein do not necessarily state or reflect those of the United States Government or the University of California, and shall not be used for advertising or product endorsement purposes.

This work was performed under the auspices of the U.S. Department of Energy by University of California, Lawrence Livermore National Laboratory under Contract W-7405-Eng-48. 


\section{Oracle Log Buffer Queueing}

Andy Rivenes

Originally investigated, December 10, 2003

(c) Lawrence Livermore National Laboratory 7000 East Avenue • L-089

Livermore, CA 94551 


\section{Table of Contents}

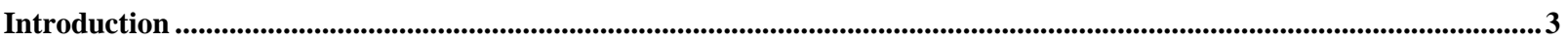

Testing Overview

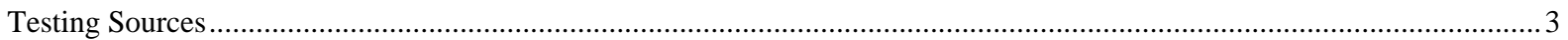

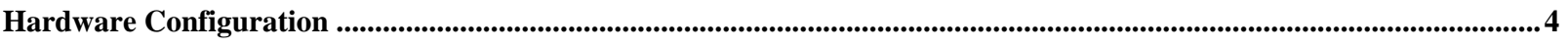

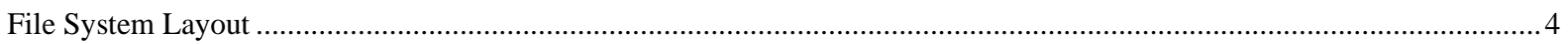

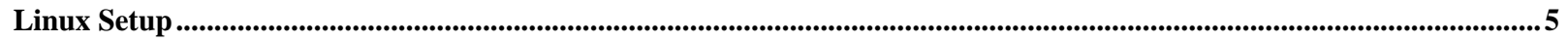

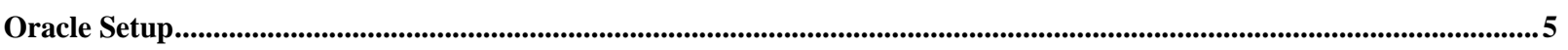

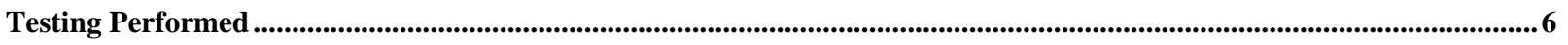

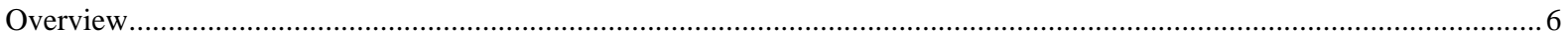

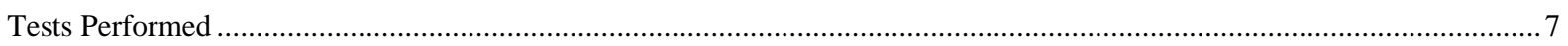

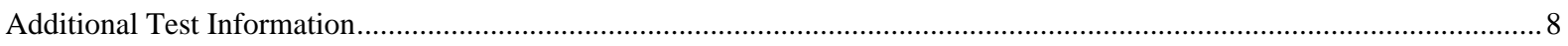

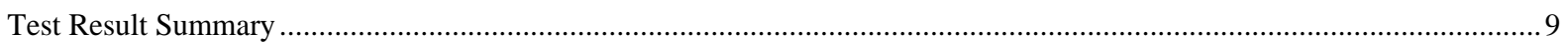

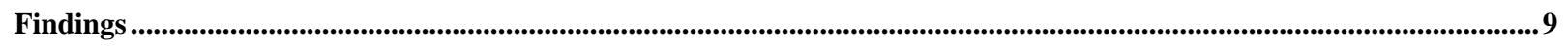

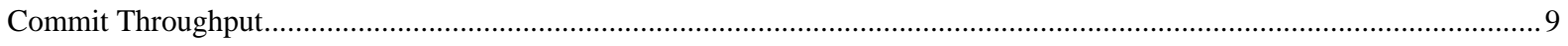

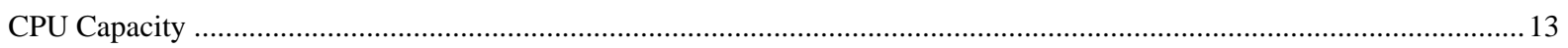

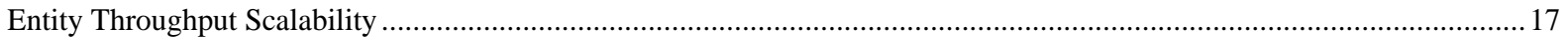

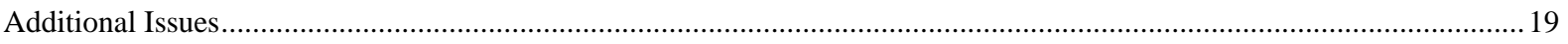

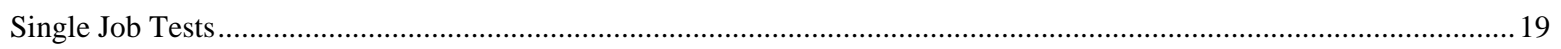

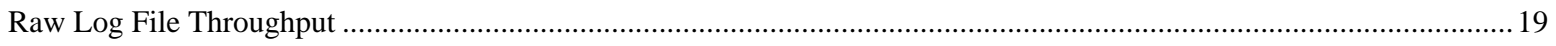

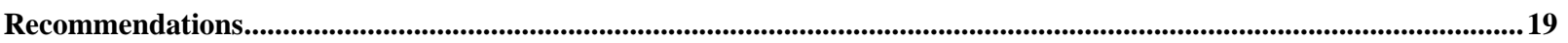

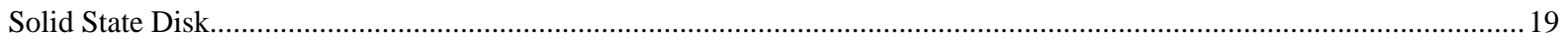

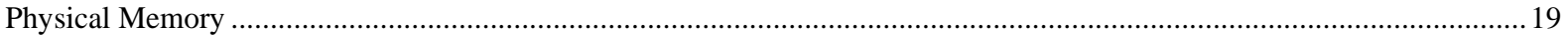

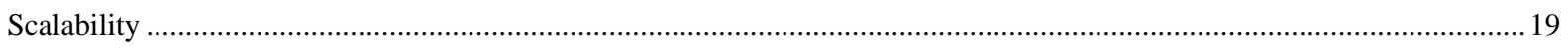

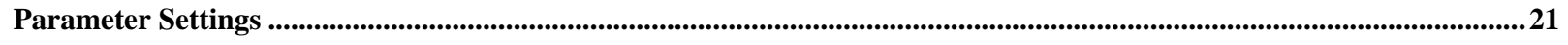

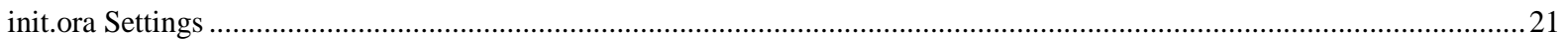

References 


\section{Introduction}

The purpose of this document is to investigate Oracle database log buffer queuing and its affect on the ability to load data using a specialized data loading system. Experiments were carried out on a Linux system using an Oracle 9.2 database. Previous experiments on a Sun 4800 running Solaris had shown that 100,000 entities per minute was an achievable rate. The question was then asked, can we do this on Linux, and where are the bottlenecks? A secondary question was also lurking, how can the loading be further scaled to handle even higher throughput requirements?

Testing was conducted using a Dell PowerEdge 6650 server with four CPUs and a Dell PowerVault 220s RAID array with 14 36GB drives and $128 \mathrm{MB}$ of cache. Oracle Enterprise Edition 9.2.0.4 was used for the database and Red Hat Linux Advanced Server 2.1 was used for the operating system. This document will detail the maximum observed throughputs using the same test suite that was used for the Sun tests. A detailed description of the testing performed along with an analysis of bottlenecks encountered will be made. Issues related to Oracle and Linux will also be detailed and some recommendations based on the findings.

\section{Testing Overview}

The testing was conducted by splitting up the data provided by each source into nine relatively equal groups. The loading code was then run for each group simultaneously. This translated into nine Java loading processes running against the Oracle database. Once all nine jobs were running at full utilization (as evidenced by "top") three simultaneous 5 minute "snapshots" were taken. The first was an extended SQL trace of one of the nine jobs, the second was a beginning snapshot using a SQL query based on loading info within the application schema, and the third was a "Statspack" beginning snapshot. Once 5 minutes had elapsed from the start of the extended SQL trace, the trace was stopped and another application snapshot and a "Statspack" snapshot were taken. In several tests a second five minute interval was analyzed, using a different load job for the extended SQL trace.

Initially all nine jobs were traced, but once it was established that all nine jobs had similar resource and execution profiles this was cut back to just one as described above. As described, one or two 5 minute intervals were observed for each test. These were conducted after initial startup and once the jobs were running at a relative "steady state". On several tests, a "late" interval observation was conducted to see if the resource or execution profile was different after a significant amount of time had passed (e.g. to attempt to insure that no caching or other phenomenon affected the early part of the test) and it was found to be consistent throughout the test run(s).

\section{Testing Sources}

Three testing sources were used during each test run. The primary test source was the capturing of extended SQL trace data for one or more of the jobs running in the test. Typically a five minute interval was captured and the resulting trace file was then profiled with the Hotsos Profiler. From that output the "Interval Resource Profile" was used to evaluate the response time components and their time contribution. Figure 1 shows the interval resource profile for one of the tests performed (e.g. test $7 \mathrm{a})$. 


\begin{tabular}{|c|c|c|c|c|c|c|}
\hline Response Time Component & & Duration & \# Calls & Avg & Min & $\operatorname{Max}$ \\
\hline CPU service & $239.660000 \mathrm{~s}$ & $78.2 \%$ & 220,524 & $0.001087 \mathrm{~s}$ & $0.000000 \mathrm{~s}$ & $3.490000 \mathrm{~s}$ \\
\hline log file sync & $38.596698 \mathrm{~s}$ & $12.6 \%$ & 13,240 & $0.002915 \mathrm{~s}$ & $0.000002 \mathrm{~s}$ & $0.039492 \mathrm{~s}$ \\
\hline unaccounted-for & $17.004281 \mathrm{~s}$ & $5.5 \%$ & & & & \\
\hline SQL*Net message from client & $4.956845 \mathrm{~s}$ & $1.6 \%$ & 390 & $0.012710 \mathrm{~s}$ & $0.000091 \mathrm{~s}$ & $0.140028 \mathrm{~s}$ \\
\hline buffer busy waits & $2.499641 \mathrm{~s}$ & $0.8 \%$ & 2,504 & $0.000998 \mathrm{~s}$ & $0.000001 \mathrm{~s}$ & $0.078763 \mathrm{~s}$ \\
\hline latch free & $1.771481 \mathrm{~s}$ & $0.6 \%$ & 490 & $0.003615 \mathrm{~s}$ & $0.000001 \mathrm{~s}$ & $0.038723 \mathrm{~s}$ \\
\hline SQL*Net more data from client & $1.484256 \mathrm{~s}$ & $0.5 \%$ & 6,933 & $0.000214 \mathrm{~s}$ & $0.000005 \mathrm{~s}$ & $0.114970 \mathrm{~s}$ \\
\hline enqueue & $0.487827 \mathrm{~s}$ & $0.2 \%$ & 255 & $0.001913 \mathrm{~s}$ & $0.000001 \mathrm{~s}$ & $0.035624 \mathrm{~s}$ \\
\hline log file switch completion & $0.023084 \mathrm{~s}$ & $0.0 \%$ & 2 & $0.011542 \mathrm{~s}$ & $0.008676 \mathrm{~s}$ & $0.014408 \mathrm{~s}$ \\
\hline SQL*Net more data to client & $0.006008 \mathrm{~s}$ & $0.0 \%$ & 39 & $0.000154 \mathrm{~s}$ & $0.000017 \mathrm{~s}$ & $0.000742 \mathrm{~s}$ \\
\hline direct path read (lob) & $0.004954 \mathrm{~s}$ & $0.0 \%$ & 585 & $0.000008 \mathrm{~s}$ & $0.000000 \mathrm{~s}$ & $0.001991 \mathrm{~s}$ \\
\hline SQL*Net message to client & $0.002308 \mathrm{~s}$ & $0.0 \%$ & 390 & $0.000006 \mathrm{~s}$ & $0.000001 \mathrm{~s}$ & $0.000033 \mathrm{~s}$ \\
\hline db file sequential read & $0.002193 \mathrm{~s}$ & $0.0 \%$ & 27 & $0.000081 \mathrm{~s}$ & $0.000038 \mathrm{~s}$ & $0.000109 \mathrm{~s}$ \\
\hline direct path write & $0.000904 \mathrm{~s}$ & $0.0 \%$ & 78 & $0.000012 \mathrm{~s}$ & $0.000001 \mathrm{~s}$ & $0.000037 \mathrm{~s}$ \\
\hline buffer deadlock & $0.000054 \mathrm{~s}$ & $0.0 \%$ & 22 & $0.000002 \mathrm{~s}$ & $0.000001 \mathrm{~s}$ & $0.000008 \mathrm{~s}$ \\
\hline otal & $4 \mathrm{~s}$ & $0 \%$ & & & & \\
\hline
\end{tabular}

Figure 1. Interval resource profile, test 7a, file 15068.

The second test source was obtained from a snapshot procedure that is part of the application schema. This information approximates the number of "entities per minute" being loaded into the database for a given time interval. The third test source was obtained from Oracle's Statspack utility. This utility captures database statistic and wait event information for a given time interval. The information used from this utility was purely statistic based and included the number of system wide user commits and redo blocks written.

\section{Hardware Configuration}

As stated in the introduction, testing was conducted on a 4-way Dell PowerEdge 6650 server with a PowerVault 220s providing disk array storage. The PowerEdge server had 4 Intel Xeon $2.5 \mathrm{GHz}$ CPUs with $1 \mathrm{MB}$ cache, 16 GB of memory, 2 SCSI interfaces, and 1 NIC.

The PowerVault 220s had $1436 \mathrm{~GB}$ drives and $128 \mathrm{MB}$ of cache. The array was divided into 3 RAID 1 volumes for a total of 6 disks, and 1 RAID 1+0 4 disk wide volume for a total of 8 disks. Initially all logical drives were set with "write-thru" caching. The RAID 1 volumes were set with $64 \mathrm{~K}$ stripe widths and the RAID $1+0$ with $128 \mathrm{~K}$ stripe width.

\section{File System Layout}

The following basic file system layout was used:

\begin{tabular}{|l|l|l|l|}
\hline $\begin{array}{l}\text { Mount } \\
\text { point }\end{array}$ & $\begin{array}{l}\text { Volume } \\
\text { type }\end{array}$ & $\begin{array}{l}\text { File } \\
\text { system }\end{array}$ & Usage \\
\hline /oracle & RAID 1 & ext3 & $\begin{array}{l}\text { used for Oracle software (e.g. ORACLE_HOME), admin } \\
\text { directories, local directories including application test data, and } \\
\text { one control file (e.g. /oracle/oradata/SID/control02.ctl) }\end{array}$ \\
\hline
\end{tabular}




\begin{tabular}{|l|l|l|l|}
\hline /ora01 & RAID 1+0 & ext3/raw & $\begin{array}{l}\text { used for Oracle database files, other two control files. Raw and } \\
\text { file system redo was tested, but not significantly different than } \\
\text { RAID 1. }\end{array}$ \\
\hline /ora02 & RAID 1 & ext3/raw & $\begin{array}{l}\text { Used for redo log files. Was used for database files when the } \\
\text { RAID } 1+0 \text { volume was used for redo log files. }\end{array}$ \\
\hline /ora03 & RAID 1 & ext3/raw & $\begin{array}{l}\text { Used for redo log files. Was used for database files when the } \\
\text { RAID 1+0 volume was used for redo log files. }\end{array}$ \\
\hline
\end{tabular}

\section{Linux Setup}

Red Hat Advanced Server 2.1 Linux was installed with the enterprise kernel (e.g. Linux 2.4.9e.3enterprise). It was initially thought that Oracle would be set up with large memory support (e.g. the target was a 12 GB SGA) and so the system was configured to allow this by using a shared memory file system (e.g. / dev/shm (shmfs)). No special kernel parameters were set other than those required by Oracle, specifically for semaphores and shared memory.

This test wasn't meant to be a comprehensive test on file system performance. However, because the resource profile of the test jobs had a large "log file sync"1 component, the redo log setup was given particular scrutiny. Based on industry information/recommendations two flavors of file systems were tested for online redo log files, raw devices and ext3 file systems. For all other database files just the ext3 file system was used.

It is worth noting that several sources were found that indicated that raw device performance was sub-optimal in Linux and that the ext3 file system performed best for Oracle databases. In addition to raw vs. ext3 file systems, asynchronous I/O was also tested. Since Linux does not support file system direct I/O (e.g. unbuffered file system I/O) that feature could not be tested.

Once the redo log file I/O was identified as a significant bottleneck we also wanted to experiment with the speed of a solid state disk device in order to see if the elimination of the log file sync bottleneck would indeed drive up CPU usage, or result in some other unforeseen bottleneck. Since we didn't have access to a solid state disk system, this was simulated using "RAM" disks. This would not be an acceptable production setup since RAM disks would not protect from an instance failure with the loss of the UNIX memory system, but they were sufficient to simulate the latency and throughput of a solid state disk.

\section{Oracle Setup}

Oracle was installed using version 9.2.0.1 and patch set 9.2.0.4. A basic database was created with the following options:

- Enterprise Edition

\footnotetext{
1 The Oracle wait event "log file sync" occurs when a user session COMMITs (or rolls back), the sessions redo information needs to be flushed to the redo logfile. The user session will post the LGWR to write all redo required from the $\log$ buffer to the redo log file. When the LGWR has finished it will post the user session. The user session waits on this wait event while waiting for LGWR to post it back to confirm all redo changes are safely on disk. - Oracle Note: 34592.1
} 
- Oracle JVM

- Oracle Spatial

- Oracle InterMedia

- Oracle Text

- Oracle XML DB

Initially the database was installed with two $1 \mathrm{~GB}$ redo $\log$ files on dedicated RAID 1 devices (e.g. /ora02 and /ora03), and all database files on a RAID 1+0 volume (e.g. /ora01). A total of three control files were used, one control file was located on the /oracle volume along with the ORACLE_HOME software and ORACLE_BASE/admin directories, and the other two were located on the /ora01 volume (e.g. RAID 1+0).

The Oracle kernel was linked with the "async_on" option and the "disk_asynch_io" init.ora parameter was set to true initially. The SGA was set to approximately $800 \mathrm{MB}$ (e.g. 400MB for database block buffers, $400 \mathrm{MB}$ for the shared pool, and $1 \mathrm{MB}$ for the log buffer). The database was created with a $4 \mathrm{~K}$ block size to match the file system block size, and no additional block sizes or buffer pools were used. The init.ora option "pga_aggregate_target" was used instead of any hash or sort area sizing. See the "Parameters" section for a list of the non-default init.ora parameters used.

\section{Testing Performed}

\section{Overview}

Several initial tests were run and in most cases the individual job resource profile consisted of $60 \%$ $70 \%$ CPU service and 10\% - 20\% "log file sync" or commit time. The rest of the time was spent among other wait events of less than $5 \%$ of total response time. From these initial tests it was clear that the loads consumed a large amount of CPU and waited a significant amount of time for commits. The actual workload consisted of a large number of inserts with additional selects and updates motivated by the inserts. In speaking with the principal developer, he concurred with this workload analysis.

In a typical response time optimization engagement, my recommendation would be to reduce the amount of CPU time and commits being performed. This follows the Hotsos Method $\mathrm{R}^{2}$ approach of observing Amdahl's law and reducing the largest time consumers in descending order. However, in speaking with the application developer, the code is complex and will be costly to modify. He is aware of the limitations and enhancements are being planned. In the meantime, our goal remained unchanged, but the code was not changeable. This drove most of the testing to find ways to reduce the commit time (e.g. the duration of the log file sync wait events) in order to maximize CPU service time and thereby load the maximum number of entities possible. As an additional exercise, I made an attempt to quantify the theoretical maximums that might be possible given additional CPUs vs. additional nodes based on the queuing characteristics of the application (e.g. arrival rate and relative service times).

2 Optimizing Oracle Performance, Pg. 20

Logbuffer_Queueing21.doc

Version 2.1, December 10, 2004 


\section{Tests Performed}

Twelve recorded tests were performed in all. The following describes the goals and results for each test:

\begin{tabular}{|c|c|c|c|}
\hline Test & Environment & Goals & Results \\
\hline 1 & $\begin{array}{l}1 \text { job test using file } \\
\text { system based redo logs }\end{array}$ & $\begin{array}{l}\text { Attempt a baseline } \\
\text { single user test and } \\
\text { establish the maximum } \\
\text { throughput for a single } \\
\text { job. }\end{array}$ & $\begin{array}{l}\text { Questionable validity. The commits per } \\
\text { CPU second are twice the number of } \\
\text { system wide commits per second that } \\
\text { was recorded by Statspack. }\end{array}$ \\
\hline 2 & $\begin{array}{l}9 \text { job test using file } \\
\text { based redo logs. }\end{array}$ & $\begin{array}{l}\text { Attempt an initial load } \\
\text { test. }\end{array}$ & $\begin{array}{l}\text { Showed that } \log \text { file sync times were } \\
\text { indeed a significant bottleneck to } \\
\text { increased throughput. }\end{array}$ \\
\hline 3 & $\begin{array}{l}1 \text { job test using RAM } \\
\text { based redo log files. }\end{array}$ & $\begin{array}{l}\text { Show the fastest } \\
\text { possible loading in the } \\
\text { absence of any } \\
\text { significant bottlenecks. }\end{array}$ & $\begin{array}{l}\text { Clearly showed that the latency of log } \\
\text { file syncs was inhibiting throughput. }\end{array}$ \\
\hline 4 & $\begin{array}{l}9 \text { job test using RAM } \\
\text { based redo log files. }\end{array}$ & $\begin{array}{l}\text { Attempt the highest } \\
\text { throughput } \\
\text { possible. }\end{array}$ & $\begin{array}{l}\text { Achieved } 100 \mathrm{~K} \text { entities per second at } \\
100 \% \text { CPU utilitization. log file sync } \\
\text { accounted for only } 5 \% \text { of individual } \\
\text { job response time and the bottleneck } \\
\text { moved to the CPUs. }\end{array}$ \\
\hline 5 & $\begin{array}{l}1 \text { job test using RAID } \\
1 \text {, file system based } \\
\text { redo logs with } \\
\text { asynchronous I/O. }\end{array}$ & $\begin{array}{l}\text { Show the highest disk } \\
\text { based throughput. }\end{array}$ & $\begin{array}{l}\text { Showed a more reasonable correlation } \\
\text { between system commit rate and } \\
\text { individual job commit rates. I felt this } \\
\text { test might have unduly benefited from } \\
\text { the array cache so a } 2 \text { and } 4 \text { job test } \\
\text { were performed later. }\end{array}$ \\
\hline 6 & $\begin{array}{l}9 \text { job test using RAID } \\
1 \text {, file system based } \\
\text { redo logs with } \\
\text { asynchronous I/O. }\end{array}$ & $\begin{array}{l}\text { Show the highest } \\
\text { loaded disk based } \\
\text { throughput. }\end{array}$ & $\begin{array}{l}\text { Achieved } 92 \mathrm{~K} \text { entities per minute. This } \\
\text { is only } 8 \% \text { less than the max. Since the } \\
\text { commit rate was the bottleneck the } \\
\text { CPUs were not driven to } 100 \% \text {. }\end{array}$ \\
\hline $7 \mathrm{a} / \mathrm{b}$ & $\begin{array}{l}9 \text { job tests using RAID } \\
1 \text {, file system based } \\
\text { redo logs with async } \\
\text { I/O disabled in Oracle. }\end{array}$ & $\begin{array}{l}\text { Show any differences } \\
\text { between synchronous } \\
\text { and asynchronous } \\
\text { I/O. }\end{array}$ & A slight improvement over async I/O. \\
\hline
\end{tabular}




\begin{tabular}{|c|c|c|c|}
\hline $7 \mathrm{c} / \mathrm{d}$ & $\begin{array}{l}9 \text { job tests using RAID } \\
1 \text {, file system based } \\
\text { redo logs with async } \\
\text { I/O disabled in Oracle } \\
\text { and the array write } \\
\text { back cache set to write } \\
\text { thru. }\end{array}$ & $\begin{array}{l}\text { Show the benefit of } \\
\text { the array cache for log } \\
\text { writes. }\end{array}$ & $\begin{array}{l}\text { Throughput was severely hampered. } \\
\text { Clearly some kind of caching provides } \\
\text { a huge benefit. }\end{array}$ \\
\hline 8 & $\begin{array}{l}9 \text { job test using RAID } \\
1 \text {, file system based } \\
\text { redo logs with } \\
\text { asynchronous I/O. }\end{array}$ & $\begin{array}{llll}\begin{array}{l}\text { Verify } \\
\text { results. }\end{array} & \text { Test } & 7 & \mathrm{a} / \mathrm{b} \\
& & \\
\end{array}$ & $\begin{array}{l}\text { Some problems were encountered } \\
\text { performing the test, but the results } \\
\text { were still impressive. The surprise was } \\
\text { that async I/O hurt throughput. }\end{array}$ \\
\hline 9 & $\begin{array}{l}9 \text { job test using RAID } \\
1 \text {, raw based redo log } \\
\text { files with async } \mathrm{I} / \mathrm{O} \text {. }\end{array}$ & $\begin{array}{l}\text { Show if raw based redo } \\
\text { logs performed better. }\end{array}$ & $\begin{array}{l}\text { Surprisingly raw redo log files } \\
\text { performed worse. Normally we would } \\
\text { expect them to be more efficient, but } \\
\text { apparently Linux's raw I/O } \\
\text { implementation is sub-optimal. }\end{array}$ \\
\hline 10 & $\begin{array}{l}9 \text { job test using RAID } \\
1 \text {, raw based redo log } \\
\text { files with synchronous } \\
\text { I/O. }\end{array}$ & $\begin{array}{l}\text { Same as Test 9, but } \\
\text { with synchronous I/O. }\end{array}$ & $\begin{array}{l}\text { There didn't appear to be a big } \\
\text { difference, but synchronous I/O did } \\
\text { appear to be worse. Since Linux does } \\
\text { support kernelized asynchronous I/O } \\
\text { this might be noteworthy if raw I/O } \\
\text { was as fast as file system I/O. }\end{array}$ \\
\hline 11 & $\begin{array}{l}2 \text { job test using RAID } \\
1 \text {, file system based } \\
\text { redo logs with async } \\
\text { I/O disabled in Oracle. }\end{array}$ & $\begin{array}{l}\text { A } 2 \text { job test to try to } \\
\text { show throughput with } \\
\text { a minimum of queuing } \\
\text { or load interference. }\end{array}$ & $\begin{array}{l}\text { Numbers were slightly slower than the } \\
1 \text { user test, but appeared to validate } \\
\text { baseline numbers. }\end{array}$ \\
\hline 12 & $\begin{array}{l}4 \text { job test using RAID } \\
1 \text {, file system based } \\
\text { redo logs with async } \\
\text { I/O disabled in Oracle. }\end{array}$ & $\begin{array}{l}\text { A } 4 \text { job test to try to } \\
\text { show throughput with } \\
\text { a some queuing. The } \\
\text { hope was to validate } \\
\text { the queuing models } \\
\text { with additional data } \\
\text { points. }\end{array}$ & $\begin{array}{l}\text { Began to show degradation. Another } \\
\text { datapoint to validate the queuing } \\
\text { influences. }\end{array}$ \\
\hline
\end{tabular}

\section{Additional Test Information}

Data files were placed on a RAID $1+0$ volume (e.g. / ora01). Initially it was thought that there would be I/O bottlenecks to the database files. As it turned out the only I/O bottleneck was with the redo $\log$ files. Tests were performed with redo $\log$ files on the RAID $1+0$ volume with both raw and ext3 file systems. Results were not significantly different than RAID 1, and given the RAID 1+0 expense, it was decided not to pursue additional testing. In fact, RAID $1+0$ is probably not necessary other than for the convenience of a large data file mount point. This application could probably make use of RAID 5 for the database files and save resources with no appreciable impact on performance. 


\section{Test Result Summary}

\begin{tabular}{|c|c|c|c|c|c|c|c|c|c|c|c|c|c|c|c|c|c|}
\hline \multicolumn{5}{|c|}{ Test Details } & \multicolumn{8}{|c|}{ Trace file } & \multicolumn{4}{|c|}{ Statspack } & \multirow{2}{*}{\begin{tabular}{|c|} 
NE Stats \\
entities/minute
\end{tabular}} \\
\hline \begin{tabular}{|l} 
Test \\
Number
\end{tabular} & Date & Start Time & Duration $(\mathrm{sec})$ & Test Description & Trace File & Commits & $\begin{array}{l}\text { CPU } \\
\text { Service }\end{array}$ & $\begin{array}{l}\text { Commits/ } \\
\text { CPU sec }\end{array}$ & \begin{tabular}{|l|} 
CPU \\
Interval \%
\end{tabular} & $\begin{array}{l}\text { Number of } \\
\text { log file } \\
\text { sync }\end{array}$ & \begin{tabular}{|l|l} 
Avg & Ic \\
duration & If \\
\end{tabular} & \begin{tabular}{|l|} 
log file \\
sync \\
Interval \% \\
\end{tabular} & \begin{tabular}{|l|} 
Statspack \\
Interval
\end{tabular} & commits/sec & Redo blks/sec & $\begin{array}{l}\text { Redo blks } \\
\text { / commit }\end{array}$ & \\
\hline 1 & 11/24/03 & $12: 32$ & 323 & 1 job, & 31973 & 18,030 & 175.82 & 102.55 & $54.5 \%$ & 18,308 & \begin{tabular}{|l|}
0.007459 \\
\end{tabular} & $42.3 \%$ & $14-15$ & 55 & $1,084.1$ & 19.7 & $13,090.91$ \\
\hline $2 a$ & $11 / 24 / 03$ & $12: 43$ & 340 & & 1098 & 13,059 & 238.44 & 54.77 & $70.2 \%$ & 12,869 & \begin{tabular}{|l|}
0.005745 \\
\end{tabular} & $21.8 \%$ & $16-17$ & 355.9 & $\frac{1,007.1}{6,697.3}$ & 18.8 & $84,449.57$ \\
\hline $2 \mathrm{~b}$ & $11 / 24 / 03$ & $12: 50$ & 364 & & 1091 & 12,566 & 236.22 & 53.20 & $64.9 \%$ & 13,236 & \begin{tabular}{|l|}
0.007934 \\
\end{tabular} & $28.8 \%$ & $17-18$ & 350.3 & $6,788.2$ & 19.4 & $83,358.78$ \\
\hline 3 & $11 / 24 / 03$ & $17: 15$ & 283 & $\begin{array}{l}1 \text { job test, redo on } \\
\text { RAM disks }\end{array}$ & 3291 & 27,593 & 272.80 & 101.15 & $96.5 \%$ & 26,783 & 0.000038 & $0.4 \%$ & $19-20$ & 97.4 & 1.917 .2 & 19.7 & $23,294.12$ \\
\hline $4 a$ & $11 / 24 / 03$ & $17: 36$ & 272 & RAM disk redo & 7107 & 10,830 & 232.97 & 46.49 & $85.9 \%$ & 9,493 & \begin{tabular}{|l|}
0.001593 \\
\end{tabular} & $5.6 \%$ & $21-22$ & 422.4 & $8,299.2$ & 19.7 & $100,751.68$ \\
\hline $4 \mathrm{~b}$ & $11 / 24 / 03$ & $17: 41$ & 270 & 2nd 5 minutes & 7097| & 10,136 & 228.40 & 44.38 & $84.8 \%$ & 8,583 & \begin{tabular}{|l|}
0.001464 \\
\end{tabular} & $4.7 \%$ & $22-23$ & 4 & $8,137.3$ & 19.9 & $98,006.69$ \\
\hline 5 & $11 / 25 / 03$ & 10:44 & 300 & 1 job test, RAID 1 , fs & 11265 & 25,117 & 246.18 & 102.03 & $82.3 \%$ & 25,624 & 0.001091 & $9.3 \%$ & $24-25$ & 83.4 & $1,639.0$ & 19.7 & Missing \\
\hline $6 \mathrm{a}$ & $11 / 25 / 03$ & 10:55 & 287 & $\begin{array}{l}\text { RAID 1 redo, fs, } \\
\text { async }\end{array}$ & 12848 & 10,658 & 227.46 & 46.86 & $79.5 \%$ & 10,030 & 0.003744 & $13.1 \%$ & $26-27$ & 389.7 & $7,557.9$ & 19.4 & $92,178.34$ \\
\hline $6 \mathrm{~b}$ & $11 / 25 / 03$ & $11: 00$ & 257 & $\begin{array}{l}\text { 2nd } 5 \text { minutes } \\
\end{array}$ & 12854 & 9,360 & 206.12 & 45.41 & $80.4 \%$ & 8,552 & \begin{tabular}{|l|}
0.003918 \\
\end{tabular} & $13.1 \%$ & $27-28$ & 382.7 & $7,424.4$ & 19.4 & $91,452.63$ \\
\hline $7 a$ & $11 / 25 / 03$ & $13: 28$ & 307 & $\begin{array}{l}\text { RAID } 1 \text { redo, fs, no } \\
\text { async }\end{array}$ & 15068 & 11,924 & 239.66 & 49.75 & $78.2 \%$ & 13,240 & 0.002915 & $12.6 \%$ & $29-30$ & 397.1 & $7,634.8$ & 19.2 & $94,390.24$ \\
\hline $7 \mathrm{~b}$ & $11 / 25 / 03$ & 13:33 & 705 & 2nd 5 minutes & 15074 & 12,822 & 272.04 & 47.13 & $38.6 \%$ & 13,864 & \begin{tabular}{|l|}
0.003116 \\
\end{tabular} & $6.1 \%$ & $30-31$ & 394.1 & $7,586.1$ & 19.3 & $93,928.93$ \\
\hline $7 \mathrm{c}$ & $11 / 25 / 03$ & 13:57 & 47 & $\begin{array}{l}\text { RAID } 1 \text { redo, fs, no } \\
\text { async, write thru on } \\
\text { array (no cache) }\end{array}$ & 17192 & 933 & 20.92 & 44.60 & $45.2 \%$ & 1,053 & 0.008425 & $19.2 \%$ & (only 1 inter & erval for both tests & & & $\begin{array}{l}\text { (only } 1 \text { interval } \\
\text { for both tests) } \\
\end{array}$ \\
\hline $7 \mathrm{~d}$ & $11 / 25 / 03$ & 13:58 & 296 & Same test as 7c & 17174 & 9,339 & 145.14 & 64.34 & $49.1 \%$ & 9,024 & \begin{tabular}{|l|}
0.012749 \\
\end{tabular} & $38.9 \%$ & $32-33$ & 297.5 & $5,509.8$ & 18.5 & $70,464.00$ \\
\hline $8 a$ & $12 / 1 / 03$ & $11: 30$ & 279 & $\begin{array}{l}\text { RAID } 1 \text { redo, fs, no } \\
\text { async }\end{array}$ & 6725 & 10,065 & 215.64 & 46.68 & $77.5 \%$ & 9,765 & 0.002878 & $10.1 \%$ & $34-35$ & 383.1 & $7,551.7$ & 19.7 & $91,722.77$ \\
\hline $8 \mathrm{~b}$ & $12 / 1 / 03$ & $11: 54$ & 111 & $\begin{array}{l}\text { Note: This was taken } \\
\text { at the end after some } \\
\text { jobs had finished! }\end{array}$ & 6727 & 5,998 & 93.79 & 63.95 & $85.0 \%$ & 5,575 & 0.001493 & $7.5 \%$ & $37-38$ & 277.8 & $5,693.6$ & 20.5 & $30,789.89$ \\
\hline$\frac{9 a}{9 b}$ & $\frac{12 / 1 / 03}{12 / 1 / 03}$ & $\frac{17: 14}{17: 18}$ & $\frac{240}{362}$ & $\begin{array}{l}\text { RAID } 1 \text { redo, raw, } \\
\text { async l/O } \\
\text { 2nd5 minutes }\end{array}$ & \begin{tabular}{|l|l}
3511 \\
3513
\end{tabular} & \begin{tabular}{r|r|}
8,648 \\
12,055
\end{tabular} & $\frac{168.10}{215.44}$ & $\frac{51.45}{55.96}$ & $\begin{array}{l}70.2 \% \\
59.6 \% \\
\end{array}$ & $\begin{array}{r}8,134 \\
13,685\end{array}$ & \begin{tabular}{|l|}
0.007014 \\
0.007986
\end{tabular} & $\frac{23.8 \%}{30.2 \%}$ & $\frac{40-41}{41-42}$ & \begin{tabular}{l|}
374.2 \\
342.1
\end{tabular} & \begin{tabular}{l|}
$7,176.9$ \\
$6,645.7$
\end{tabular} & $\frac{19.2}{19.4}$ & $\begin{array}{r}89,727.27 \\
81,391.30\end{array}$ \\
\hline $10 \mathrm{a}$ & $12 / 1 / 03$ & 18:01 & 305 & \begin{tabular}{|l|} 
RAID 1 redo, raw, no \\
async
\end{tabular} & 6875 & 11,173 & 203.92 & 54.79 & $66.9 \%$ & 11,518 & 0.007162 & $27.1 \%$ & $\frac{43-44}{44-45}$ & $\frac{368.9}{334.9}$ & $6,955.4$ & 18.9 & $87,662.34$ \\
\hline $10 \mathrm{~b}$ & $12 / 1 / 03$ & 18:06 & 394 & & 6883 & 14,076 & 256.31 & 54.92 & $64.9 \%$ & 12,386 & \begin{tabular}{|l|}
0.007419 \\
\end{tabular} & $23.3 \%$ & $44-45$ & 334.6 & $6,358.9$ & 19.0 & $79,534.88$ \\
\hline $11 \mathrm{a}$ & $12 / 2 / 03$ & $16: 48$ & 24 & $\begin{array}{l}2 \text { job test, RAID } 1 \\
\text { redo, fs, no async }\end{array}$ & 3347 & 1,996 & 20.83 & 95.82 & $86.3 \%$ & 2,340 & 0.001393 & $13.5 \%$ & $46-47$ & 176.8 & $3,412.60$ & 19.3 & $41,907.12$ \\
\hline $11 \mathrm{~b}$ & $12 / 2 / 03$ & $16: 43$ & 299 & $\begin{array}{l}2 \text { job test, RAID } 1 \\
\text { redo, fs, no async }\end{array}$ & 3349 & 24,075 & 262.64 & 91.67 & $87.9 \%$ & 21,767 & 0.001286 & $9.4 \%$ & $47-48$ & 138.9 & $2,709.70$ & 19.5 & $33,237.21$ \\
\hline $12 \mathrm{a}$ & $12 / 2 / 03$ & 17:01 & 271 & $\begin{array}{l}4 \text { job test, RAID } 1 \\
\text { redo, fs, no async }\end{array}$ & 4871 & 16,752 & 224.44 & 74.64 & $83.0 \%$ & 18,550 & 0.001941 & $13.3 \%$ & $49-50$ & 276.6 & $5,299.40$ & 19.2 & $65,981.42$ \\
\hline $12 b$ & $12 / 2 / 03$ & $16 \cdot 56$ & 296 & $\begin{array}{l}4 \text { job test, RAID } 1 \\
\text { redo, fs, no async }\end{array}$ & 4874 & 17.041 & 239.45 & 71.17 & $81.1 \%$ & 17.463 & 0.002376 & $14.0 \%$ & $50-51$ & 265.9 & $5,100.10$ & 19.2 & $63,476.29$ \\
\hline
\end{tabular}

\section{Findings}

\section{Commit Throughput}

Based on initial tests performed, the main testing emphasis was placed on reducing commit time and therefore increasing commit throughput. The individual job average duration times for log file sync varied with load, and I believe this was due primarily to queuing and the caching constraints in the RAID array. The best fully loaded log file sync times achieved with disk devices was 2.9 milliseconds for average duration. At the system measurement level this translated to 2.5 milliseconds. I believe this slight discrepancy can be explained if we take in to account a slight overlap in I/Os since there were 9 independent jobs running. While it is true that this test did not take advantage of asynchronous I/O (and was still faster), if we look at the asynchronous I/O tests we see an even greater disparity between individual job duration times and overall system throughput (e.g. 3.7 milliseconds vs. 2.6 milliseconds). My best guess is that for LGWR writes the asynchronous code path consumes more time than performing the writes synchronously.

By far the best throughput was obtained using ram disks for redo logging (e.g. tests 3 and 4). This allowed the CPU utilization of the machine to approach 100\% and the individual job response time component to approach 86\% for CPU service. See Figure 2 for the queuing reduction differences. 
In terms of redo configuration, it appears that RAID 1 devices, with the $128 \mathrm{MB}$ of RAID array cache provided by the PowerVault 220s, and mounted as ext3 file systems with synchronous I/O are capable of supporting a maximum of about 400 commits/sec. This translates into an average service time of about 2.5 milliseconds. This results in an effective load rate of approximately $94 \mathrm{~K}$ entities/minute. At this rate the CPUs are not at full utilization and the system is achieving approximately a $94 \%$ utilization rate 3 . See test 7 for these details. In order to achieve the additional $6 \%$ utilization it will require redo logging to solid state disk 4 . This will reduce the average duration of a "log file sync" low enough to reduce the queueing effects at the necessary arrival rate. If the "log file sync" service rate is not reduced, then adding more CPUs will only make the problem worse, not better. Figure 3 illustrates this phenomenon. If additional CPUs are added, the arrival rate of new work will move the system to the far right of the performance curve knee. The ability to meet the response time goal moves from $60 \%$ satisfaction to only $28 \%$ satisfaction. The reason our response time goal is only $60 \%$ satisfaction is that I based the response time on a commit rate to achieve $100 \mathrm{~K}$ entities per minute. Since we can only achieve this with solid state disk, even our "best" disk solution cannot satisfy our response time goal.

In order to increase the single instance commit throughput, the log file sync service time must be reduced below a 2.5 milliseconds average service rate.

${ }^{3}$ This figure was derived by using the maximum rate obtained with RAM disk redo logs as the maximum rate possible (e.g. $100 \%$ utilization).

${ }_{4}^{4}$ Clearly using RAM disks is not an acceptable production solution. Since RAM disks reside in volatile system memory they cannot preserve the online redo $\log$ files in the event of a memory or machine failure and therefore we cannot guarantee recovery from instance failure. 


\section{Queueing Theory Multiserver Model}

$\mathrm{M} / \mathrm{M} / \mathrm{m} 3.1 \mathrm{e}(2003 / 03 / 11)$

Copyright (C) 1999-2003 by Hotsos Enterprises, Ltd. All rights reserved

\begin{tabular}{|c|c|c|c|}
\hline name & value $_{a}$ & value $_{b}$ & description \\
\hline \multicolumn{4}{|c|}{ Units of measure } \\
\hline jobunit & & commit & workload unit (singular) \\
\hline timeunit & & sec & time unit (singular) \\
\hline queueunit & & instance & queue unit (singular) \\
\hline serverunit & & log file sync & service channel unit (singular) \\
\hline serviceunit & & sec/commit & service unit \\
\hline throughputunit & & commit/sec & throughput unit \\
\hline \multicolumn{4}{|c|}{ Service level agreements } \\
\hline$\lambda$ & 397 & 422 commit/sec & average arrival rate into the system \\
\hline $\begin{array}{l}r_{\max } \\
\text { Architecture }\end{array}$ & 0.0025 & $0.0025 \mathrm{sec} / \mathrm{commit}$ & maximum tolerated response time \\
\hline$q$ & 1 & 1 instance & number of instances \\
\hline$m$ & 1 & 1 log file sync/inst & number of log file syncs per instance \\
\hline$\mu$ & 769 & $1050 \mathrm{commit} / \mathrm{sec}$ & average service rate \\
\hline \multicolumn{4}{|c|}{ Performance forecasts } \\
\hline color code & $\bullet$ & - & graph color and shape code \\
\hline model & $1 \times M / M / 1$ & $1 \times M / M / 1$ & Kendall notation \\
\hline$\rho$ & $51.6 \%$ & $40.2 \%$ & average utilization per log file sync \\
\hline$S$ & 0.001300 & $0.000952 \mathrm{sec} / \mathrm{commit}$ & average service time \\
\hline$w$ & 0.001387 & $0.000640 \mathrm{sec} / \mathrm{commit}$ & average queueing delay at specified $\lambda$ \\
\hline$R$ & 0.002687 & $0.001592 \mathrm{sec} / \mathrm{commit}$ & average response time at specified $\lambda$ \\
\hline$C D F\left(r_{\max }\right)$ & $60.567 \%$ & $79.195 \%$ satisfactions & $\%$ of jobs with $R \leq r_{\max }$ at specified $\lambda$ \\
\hline $1-C D F\left(r_{\max }\right)$ & $39.433 \%$ & $\mathbf{2 0 . 8 0 5 \%}$ dissatisfactions & $\%$ of jobs with $R>r_{\max }$ at specified $\lambda$ \\
\hline
\end{tabular}

Graph parameters

$\lambda_{0}$

$\lambda_{1} \max \left(\lambda_{\mathrm{a}}, \lambda_{\mathrm{b}}\right)$

$\lambda_{1}$

$\lambda_{\Delta}$
0 commit/sec

1.2

$506.4 \mathrm{commit} / \mathrm{sec}$

$25.32 \mathrm{commit} / \mathrm{sec}$ arrival rate axis minimum value ratio of $\lambda_{1}$ to $\max \left(\lambda_{\mathrm{a}}, \lambda_{\mathrm{b}}\right)$ arrival rate axis maximum value average arrival rate increment

Response Time $=$ Service Time + Queueing Delay

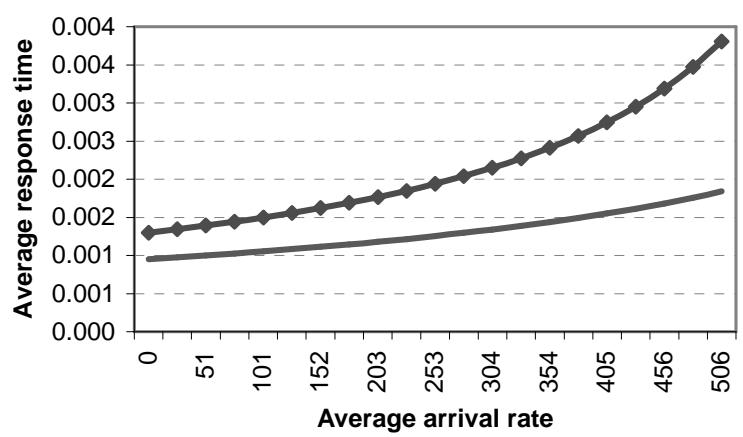

Figure 2. Redo logs on RAID 1, ext3 file system, synchronous I/O vs. RAM disks 


\section{Queueing Theory Multiserver Model}

$\mathrm{M} / \mathrm{M} / \mathrm{m} 3.1 \mathrm{e}(2003 / 03 / 11)$

Copyright (c) 1999-2003 by Hotsos Enterprises, Ltd. All rights reserved

\begin{tabular}{|c|c|c|c|}
\hline name & value $_{\mathrm{a}}$ & value $_{b}$ & description \\
\hline \multicolumn{4}{|c|}{ Units of measure } \\
\hline jobunit & & commit & workload unit (singular) \\
\hline timeunit & & sec & time unit (singular) \\
\hline queueunit & & instance & queue unit (singular) \\
\hline serverunit & & log file sync & service channel unit (singular) \\
\hline serviceunit & & sec/commit & service unit \\
\hline throughputunit & & commit/sec & throughput unit \\
\hline \multicolumn{4}{|c|}{ Service level agreements } \\
\hline$\lambda$ & 397 & 636 commit/sec & average arrival rate into the sys \\
\hline $\begin{array}{l}r_{\max } \\
\text { Architecture }\end{array}$ & 0.0025 & $0.0025 \mathrm{sec} / \mathrm{commit}$ & maximum tolerated response tin \\
\hline$q$ & 1 & 1 instance & number of instances \\
\hline$m$ & 1 & 1 log file sync/inst; & number of log file syncs per inst \\
\hline$\mu$ & 769 & 769 commit/sec & average service rate \\
\hline \multicolumn{4}{|c|}{ Performance forecasts } \\
\hline color code & & - & graph color and shape code \\
\hline model & $1 \times M / M / 1$ & $1 \times M / M / 1$ & Kendall notation \\
\hline$\rho$ & $51.6 \%$ & $82.7 \%$ & average utilization per log file sy \\
\hline$S$ & 0.001300 & $0.001300 \mathrm{sec} / \mathrm{commit}$ & average service time \\
\hline$W$ & 0.001388 & $0.006218 \mathrm{sec} / \mathrm{commit}$ & average queueing delay at speci \\
\hline$R$ & 0.002688 & $0.007519 \mathrm{sec} / \mathrm{commit}$ & average response time at specif \\
\hline$C D F\left(r_{\max }\right)$ & $60.545 \%$ & $28.287 \%$ satisfactions & $\%$ of jobs with $R \leq r_{\max }$ at speci \\
\hline $1-C D F\left(r_{\max }\right)$ & $39.455 \%$ & 71.713\% dissatisfactions & $\%$ of jobs with $R>r_{\max }$ at spec \\
\hline \multicolumn{4}{|c|}{ Graph parameters } \\
\hline$\lambda_{0}$ & & 0 commit/sec & arrival rate axis minimum value \\
\hline$\lambda_{1} \max \left(\lambda_{\mathrm{a}}, \lambda_{\mathrm{b}}\right)$ & & 1.2 & ratio of $\lambda_{1}$ to $\max \left(\lambda_{\mathrm{a}}, \lambda_{\mathrm{b}}\right)$ \\
\hline$\lambda_{1}$ & & $763.2 \mathrm{commit} / \mathrm{sec}$ & arrival rate axis maximum value \\
\hline$\lambda_{\Delta}$ & & $38.16 \mathrm{commit} / \mathrm{sec}$ & average arrival rate increment \\
\hline
\end{tabular}

Response Time $=$ Service Time + Queueing Delay

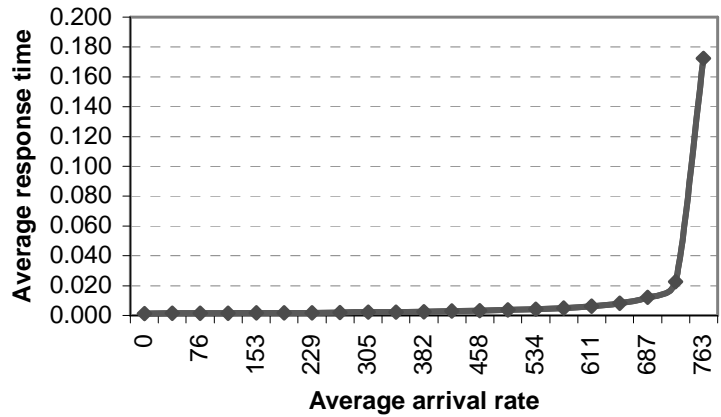

Figure 3. Commit throughput with increased workload. 


\section{CPU Capacity}

The testing also allowed some modeling of the theoretical maximums that could be expected from both commit throughput and total CPU utilization based on the given configuration. From this modeling, and the corresponding testing results, it would appear that the maximum possible throughput is about 100,000 entities per minute for this four CPU machine. In achieving this rate the CPUs are at their maximum utilization and the queuing of requests passes the "knee" 5 in the performance curve. See Figure 4 and the results of Test 7 a.

It would appear though, that with a low enough service time for commits (e.g. using a solid state disk) that a single system should continue to scale with the addition of more CPUs. Figure 5 illustrates the queuing affects of adding four additional physical processors (e.g. 8 logical processors for a total of 16 CPUs in the model) if we assume that the system can support the additional commit rate. The arrival rate of 423 was chosen since this is the number of commits per second that was observed at our peak transaction rate of $100 \mathrm{~K}$ entities/second. We see that if we double the number of CPUs that we should be able to roughly double the number of commits per second to 846 and therefore double the number entities per second that we can process. This of course ignores any other bottlenecks that might show up as a result of the addition of more workload, but this option may be more cost effective than other alternatives, and could help offset the cost of a solid state disk device.

As a secondary note, the service rate of 69 commits per second was derived from the system wide commits per second statistic obtained from Statspack. This value, when divided by the number of jobs, provides an approximation of the commits per second per CPU occurring during the test(s). As the number of jobs goes up, this value begins to go down, presumably due to the effects of queueing. In the tests conducted this value didn't really change much between a 2 and 4 job test. In the 9 job tests it did. If we follow the response time curve in Figure 3 however, this is not an unexpected result. In the case of the value of 69 , this is the value obtained from test $11 \mathrm{~b}^{6}$, which was the longer duration, and therefore probably more accurate, statistic collection for the 2 job test. Notice that this is approximately the same value that is obtained from test $12 \mathrm{a}$, and close to $12 \mathrm{~b}$, which were taken from the 4 job test.

The question could be asked, how accurate is this? Can we really equate one of the load jobs to one CPU's utilization? I believe the answer is yes, and the reasoning is fairly simple. Oracle "background" processes (e.g. the server process for application connections) are single threaded. In the absence of $\mathrm{I} / \mathrm{O}$, network, or internal database contention, an Oracle process will consume service time on a single CPU. This is fairly simple to prove, and was observed during the testing. Single job tests consumed roughly $80 \%$ of one CPU. This was verified using "top", sar and vmstat. In addition, the run queue, as evidenced in sar and top was approximately 1. In other words, there was always one process running. Occasionally a background process or the process doing the monitoring would slip in, but from the operating system's perspective there was one process running consuming most of one CPU. This was true for the 2 and 4 job tests as well, with load and run queues corresponding to the number of jobs running. This is why I chose 9 jobs for the full load test. My expectation was

5 Optimizing Oracle Performance, Pg. 257

${ }^{6}$ See Test Results for test details

Logbuffer_Queueing21.doc

Version 2.1, December 10, 2004

Page 13 
that to maximize throughput there should be some CPU queuing, and therefore CPU saturation, if we could make the jobs efficient enough to be CPU bound. In fact, Oracle helped me confirm this CPU saturation once we switched to RAM disks for the redo log files. One of the reasons that extended SQL tracing was used as one of the test sources is that Oracle records all time used in a session. In fact, it will record wall clock, or elapsed time as well. In the case of CPU saturation however, there will be a difference between the elapsed time and the recorded time of the actions performed in the session. In fact, the elapsed time will be greater than the Oracle "response" time. This is due to the fact that Oracle does not perform timings unless it is running on a CPU or waiting on some event. In the case of a process waiting to run on the operating system CPU queue, there will be a discrepancy between the elapsed, or wall clock time, which will still be measuring time, and the timings Oracle has made for the sessions actions. This is called "unaccounted-for" time by Oracle, and others in the industry. In our case, the 9 job test with redo on RAM did in fact experience unaccounted-for time. It experienced at least $3.8 \%$ of unaccounted-for time during the test. See figure 6 for the details from the 7107 trace file. 


\section{Queueing Theory Multiserver Model}

$\mathrm{M} / \mathrm{M} / \mathrm{m} 3.1 \mathrm{e}(2003 / 03 / 11)$

Copyright (c) $1999-2003$ by Hotsos Enterprises, Ltd. All rights reserved

\begin{tabular}{|c|c|c|c|}
\hline name & value $_{a}$ & value $_{b}$ & description \\
\hline \multicolumn{4}{|c|}{ Units of measure } \\
\hline jobunit & & commits & workload unit (singular) \\
\hline timeunit & & sec & time unit (singular) \\
\hline queueunit & & system & queue unit (singular) \\
\hline serverunit & & CPU & service channel unit (singular) \\
\hline serviceunit & & sec/commits & service unit \\
\hline throughputunit & & commits/sec & throughput unit \\
\hline \multicolumn{4}{|c|}{ Service level agreements } \\
\hline$\lambda$ & 97.4 & 397.1 commits/sec & average arrival rate into the system \\
\hline$r_{\max }$ & 0.015 & $0.015 \mathrm{sec} / \mathrm{commits}$ & maximum tolerated response time \\
\hline \multicolumn{4}{|l|}{ Architecture } \\
\hline$q$ & 1 & 1 system & number of systems \\
\hline$m$ & 8 & 8 CPU/system & number of CPUs per system \\
\hline$\mu$ & 69 & 69 commits/sec & average service rate \\
\hline \multicolumn{4}{|c|}{ Performance forecasts } \\
\hline color code & $\diamond$ & 一 & graph color and shape code \\
\hline model & $1 \times \mathrm{M} / \mathrm{M} / 8$ & $1 \times M / M / 8$ & Kendall notation \\
\hline$\rho$ & $17.6 \%$ & $71.9 \%$ & average utilization per CPU \\
\hline$S$ & 0.014493 & $0.014493 \mathrm{sec} / \mathrm{commits}$ & average service time \\
\hline$W$ & 0.000000 & $0.001952 \mathrm{sec} / \mathrm{commits}$ & average queueing delay at specified $\lambda$ \\
\hline$R$ & 0.014493 & $0.016445 \mathrm{sec} / \mathrm{commits}$ & average response time at specified $\lambda$ \\
\hline$C D F\left(r_{\max }\right)$ & $64.477 \%$ & $58.227 \%$ satisfactions & $\%$ of jobs with $R \leq r_{\max }$ at specified $\lambda$ \\
\hline $1-C D F\left(r_{\max }\right)$ & $35.523 \%$ & $41.773 \%$ dissatisfactions & $\%$ of jobs with $R>r_{\max }$ at specified $\lambda$ \\
\hline
\end{tabular}

\section{Graph parameters}

$\lambda_{0}$

$\lambda_{1} \max \left(\lambda_{\mathrm{a}}, \lambda_{\mathrm{b}}\right)$

$\lambda_{1}$

$\lambda_{\Delta}$
0 commits/sec

1.2

476.52 commits/sec

23.826 commits/sec

arrival rate axis minimum value

ratio of $\lambda_{1}$ to $\max \left(\lambda_{\mathrm{a}}, \lambda_{\mathrm{b}}\right)$

arrival rate axis maximum value

average arrival rate increment

Response Time $=$ Service Time + Queueing Delay

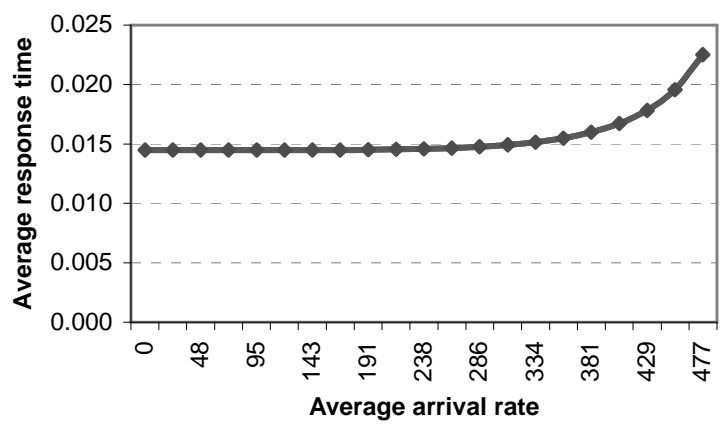

Figure 4 - CPU M/M/m queuing model 


\section{Queueing Theory Multiserver Model}

$\mathrm{M} / \mathrm{M} / \mathrm{m} 3.1 \mathrm{e}(2003 / 03 / 11)$

Copyright (c) 1999-2003 by Hotsos Enterprises, Ltd. All rights reserved

\begin{tabular}{|c|c|c|c|}
\hline name & value $_{a}$ & value $_{b}$ & description \\
\hline \multicolumn{4}{|l|}{ Units of measure } \\
\hline jobunit & & commits & workload unit (singular) \\
\hline timeunit & & $\mathrm{sec}$ & time unit (singular) \\
\hline queueunit & & system & queue unit (singular) \\
\hline serverunit & & CPU & service channel unit (singular) \\
\hline serviceunit & & sec/commits & service unit \\
\hline throughputunit & & commits/sec & throughput unit \\
\hline \multicolumn{4}{|c|}{ Service level agreements } \\
\hline$\lambda$ & 423 & 846 commits/sec & average arrival rate into the system \\
\hline \multicolumn{4}{|l|}{ Architecture } \\
\hline$q$ & 1 & 1 system & number of systems \\
\hline$m$ & 8 & 16 CPU/system & number of CPUs per system \\
\hline$\mu$ & 69 & 69 commits/sec & average service rate \\
\hline \multicolumn{4}{|c|}{ Performance forecasts } \\
\hline color code & $\bullet$ & - & graph color and shape code \\
\hline model & $1 \times M / M / 8$ & $1 \times M / M / 16$ & Kendall notation \\
\hline$\rho$ & $76.6 \%$ & $76.6 \%$ & average utilization per CPU \\
\hline$S$ & 0.014493 & $0.014493 \mathrm{sec} / \mathrm{commits}$ & average service time \\
\hline$W$ & 0.003010 & $0.000908 \mathrm{sec} / \mathrm{commits}$ & average queueing delay at specified $\lambda$ \\
\hline$R$ & 0.017502 & $0.015401 \mathrm{sec} / \mathrm{commits}$ & average response time at specified $\lambda$ \\
\hline$C D F\left(r_{\max }\right)$ & $55.066 \%$ & 61.617\% satisfactions & $\%$ of jobs with $R \leq r_{\max }$ at specified $\lambda$ \\
\hline $1-C D F\left(r_{\max }\right)$ & $44.934 \%$ & 38.383\% dissatisfactions & $\%$ of jobs with $R>r_{\max }$ at specified $\lambda$ \\
\hline
\end{tabular}

Graph parameters

$\lambda_{0}$

$\lambda_{1} \max \left(\lambda_{\mathrm{a}}, \lambda_{\mathrm{b}}\right)$

$\lambda_{1}$

$\lambda_{\Delta}$
0 commits/sec

1.2

1015.2 commits/sec

50.76 commits/sec arrival rate axis minimum value

ratio of $\lambda_{1}$ to $\max \left(\lambda_{\mathrm{a}}, \lambda_{\mathrm{b}}\right)$

arrival rate axis maximum value

average arrival rate increment

Response Time $=$ Service Time + Queueing Delay

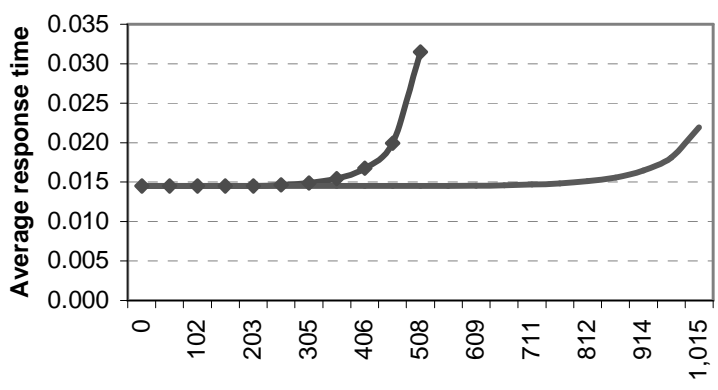

Average arrival rate

Figure 5. The effects of adding 4 more physical CPUs (total of 16 logical processors) 


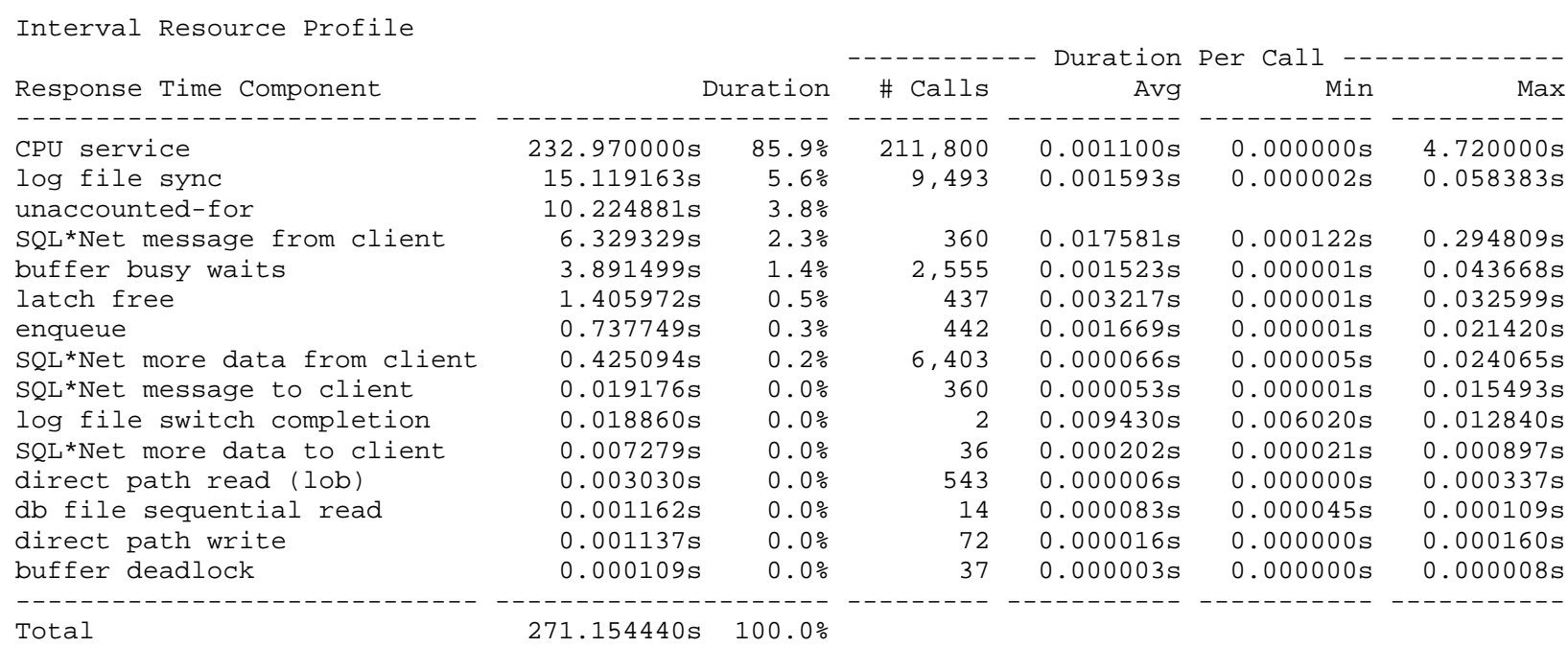

Figure 6. Resource Profile showing unaccounted-for time.

\section{Entity Throughput Scalability}

As a final wrap up on scalability, two more questions come to mind. How was the entity per minute rate affected as jobs were added, and how was the commit scalability affected? This might affect our decision on how many CPUs to place in each server. Based on the test result data from the tests with redo logs on RAID 1 volumes with ext3 file systems, synchronous I/O and write back cache enabled on the array, I plotted the entities per minute and the commits per CPU per job for 2, 4 and 9 job tests (e.g. the blue bars). See figures 7 and 8. The results show that it takes only 4 jobs to load $66 \mathrm{~K}$ entities / minute, but over twice that to get to $94 \mathrm{~K}$. The scalability is linear between 2 and 4 jobs, but really falls off at 9 . Unfortunately I should have run a 6 job test as well as an 8 job test to better pinpoint queuing affects at higher load levels (e.g. the knee of the performance curve). By adding the 9 job redo on RAM disk data we still see a large drop off (e.g. the "9-RAM" bar). At 9 jobs we know we were running to the right of the performance curve for commit rates for disk based redo and CPU queuing for RAM based redo. 


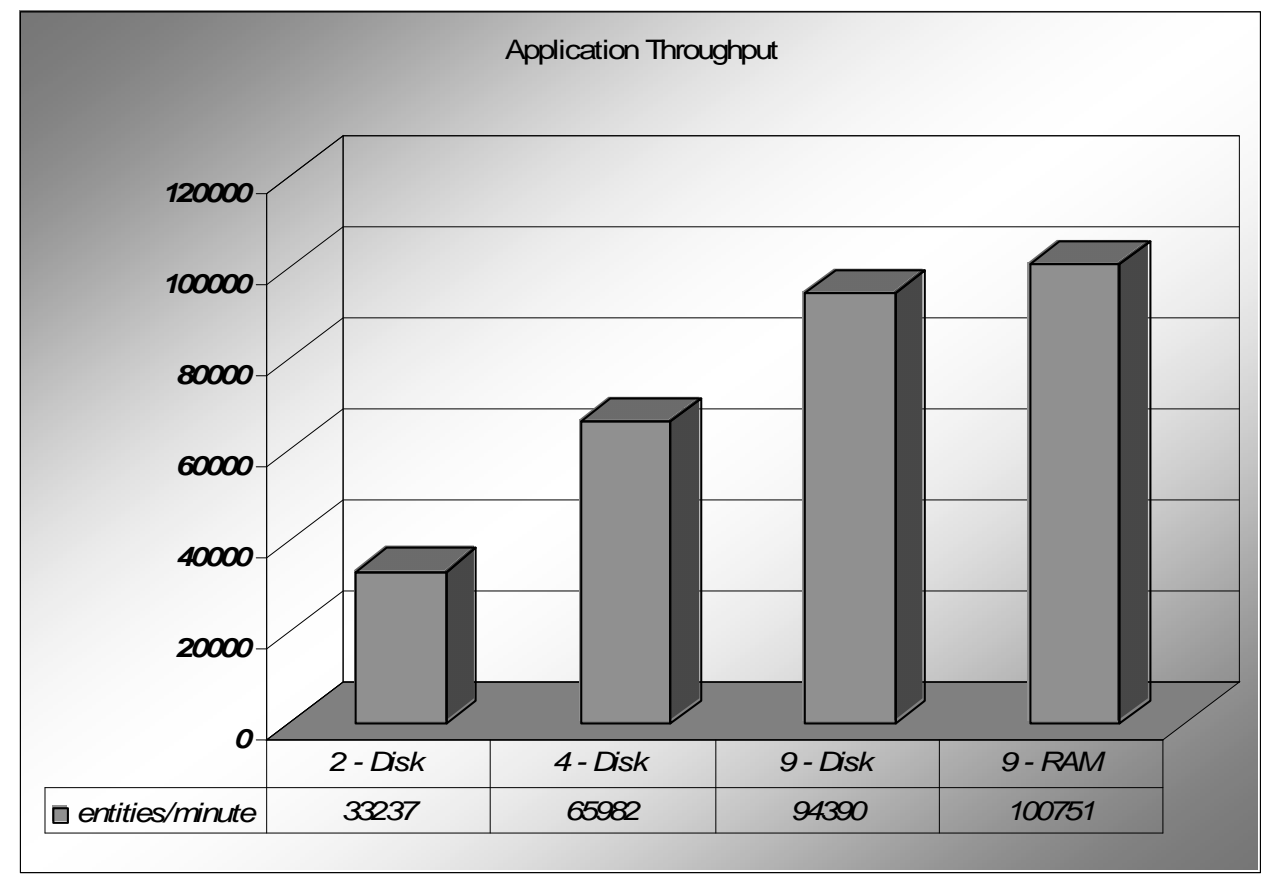

Figure 7. Entity throughput by number of jobs

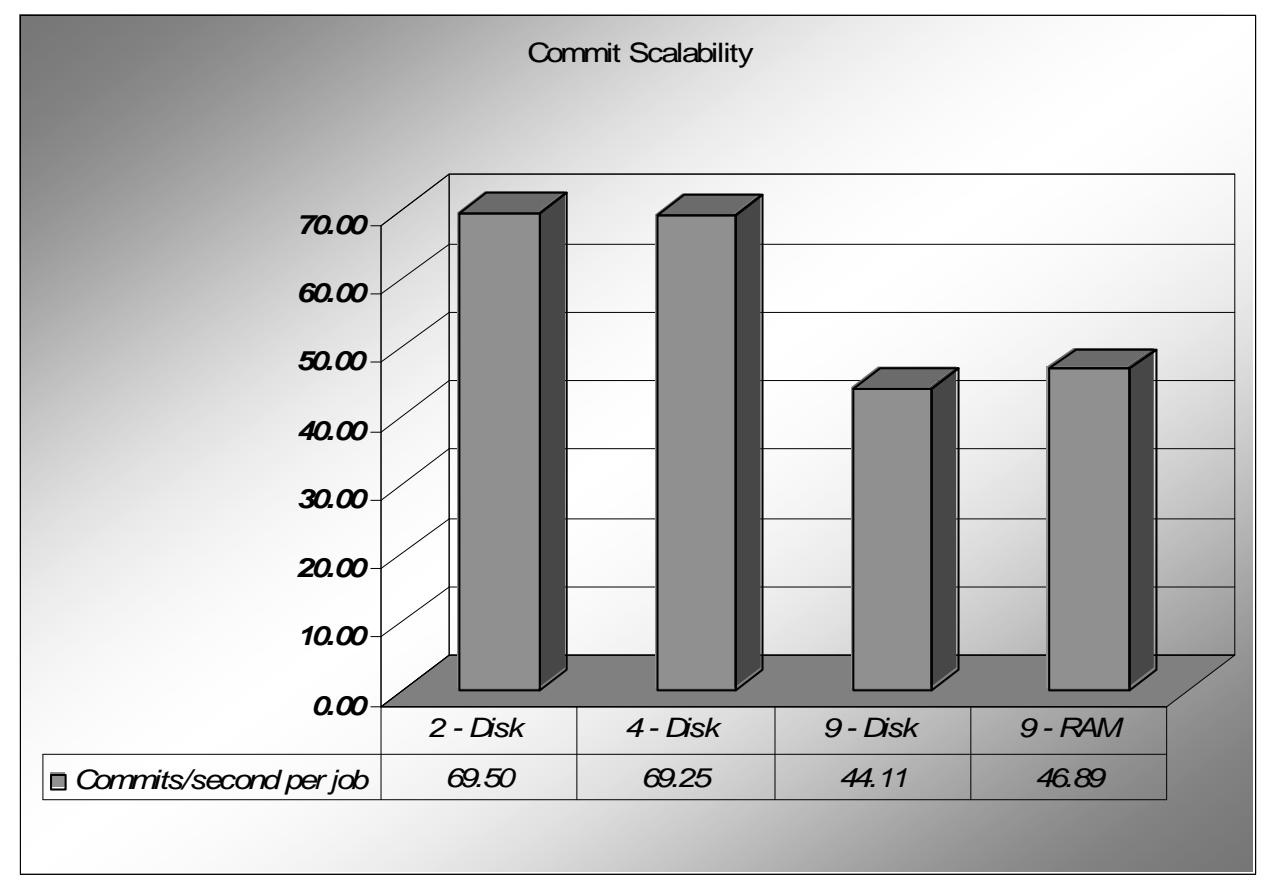

Figure 8. Commits per second per CPU 


\section{Additional Issues}

\section{Single Job Tests}

A couple of anomalies occurred during testing that I don't have answers for. The first was the issue with one job tests. In all of the one job tests, commit rates were twice what two job tests were. Based on the resource profiles for the single vs. two job tests, I do not believe that this can be explained as contention within the database. Something else was happening that I haven't figured out. Interestingly enough, all the other tests seem to fall pretty much where you would expect in relation to each other (e.g. 2, 4, 9 jobs tests).

\section{Raw Log File Throughput}

In most UNIX systems, raw log file throughput will exceed that of file system throughput. Specifically, buffered file system throughput. This is due to the fact that Oracle writes redo log files in operating size blocks rather than file system sized blocks. In Linux this means that Oracle writes 512 byte blocks instead of $4 \mathrm{~K}$ blocks. Normally, Oracle does not write 8512 byte blocks every time it writes to the redo log files. During the tests performed, the average write size was twenty 512 byte blocks which means that Oracle was writing 2.5 file system size blocks every time a commit occurred. Since the operating system can't write half a block, it has to read in the third block in this case, modify the data, and write it back out. With a raw file the operating system just writes 20 blocks. Normally the other advantage of raw file I/O is that the operating system can make use of kernelized asynchronous I/O. Interestingly enough, none of this applied in the tests conducted, and synchronous file system I/O to the redo log files was the hands down performance winner.

\section{Recommendations}

\section{Solid State Disk}

Based on the workload and resource profile of the load jobs, using a solid state disk device for the online redo log files increases the overall system throughput. During testing, the use of RAM disks (e.g. simulated SSD) reduced the "log file sync" bottleneck considerably and provided a $6 \%$ boost in entity per minute throughput. In the long term this will also "deheat" the storage array by removing approximately half the total $\mathrm{I} / \mathrm{O}$, and this is foreground $\mathrm{I} / \mathrm{O}$ (e.g. I/O that processes are directly waiting for).

\section{Physical Memory}

The machine had 16 GB of memory. Oracle used approximately 1 GB of that memory. The rest was used by processes, the OS, and the large majority by the UNIX file system cache. For this type of workload this could be scaled back significantly. I would expect that 4 GB would be sufficient.

\section{Scalability}

As part of the initial scope of this project, the question of what it would take to scale to even higher throughput requirements was asked. I believe that there are a couple of answers or guidelines that 
could be followed. The first is that each server should have as many CPUs, and therefore load jobs on an approximate one to one basis, as the commit service rate can support. In our examples, this would be approximately 8 CPUs using disk based log files that can support an approximate service time of $2.5 \mathrm{~ms}$. Even better, if solid state disk is used then even more CPUs/jobs could be supported and in the tested configuration all of the CPU capacity could be maximized.

In order to scale above the $94 \mathrm{~K}-100 \mathrm{~K}$ entity/minute rate, the next logical step would be to try Oracle's Real Application Cluster (RAC) technology. Since the bottleneck(s) revolve around the commit rate and CPU service rate, and since these are instance wide limitations within Oracle, it makes since that adding additional instances should add additional scalability. At some point other bottlenecks will probably occur, but certainly this should allow for the additional load rate scaling of a single database loader. 


\section{Parameter Settings}

\section{init.ora Settings}

System parameters with non-default values:

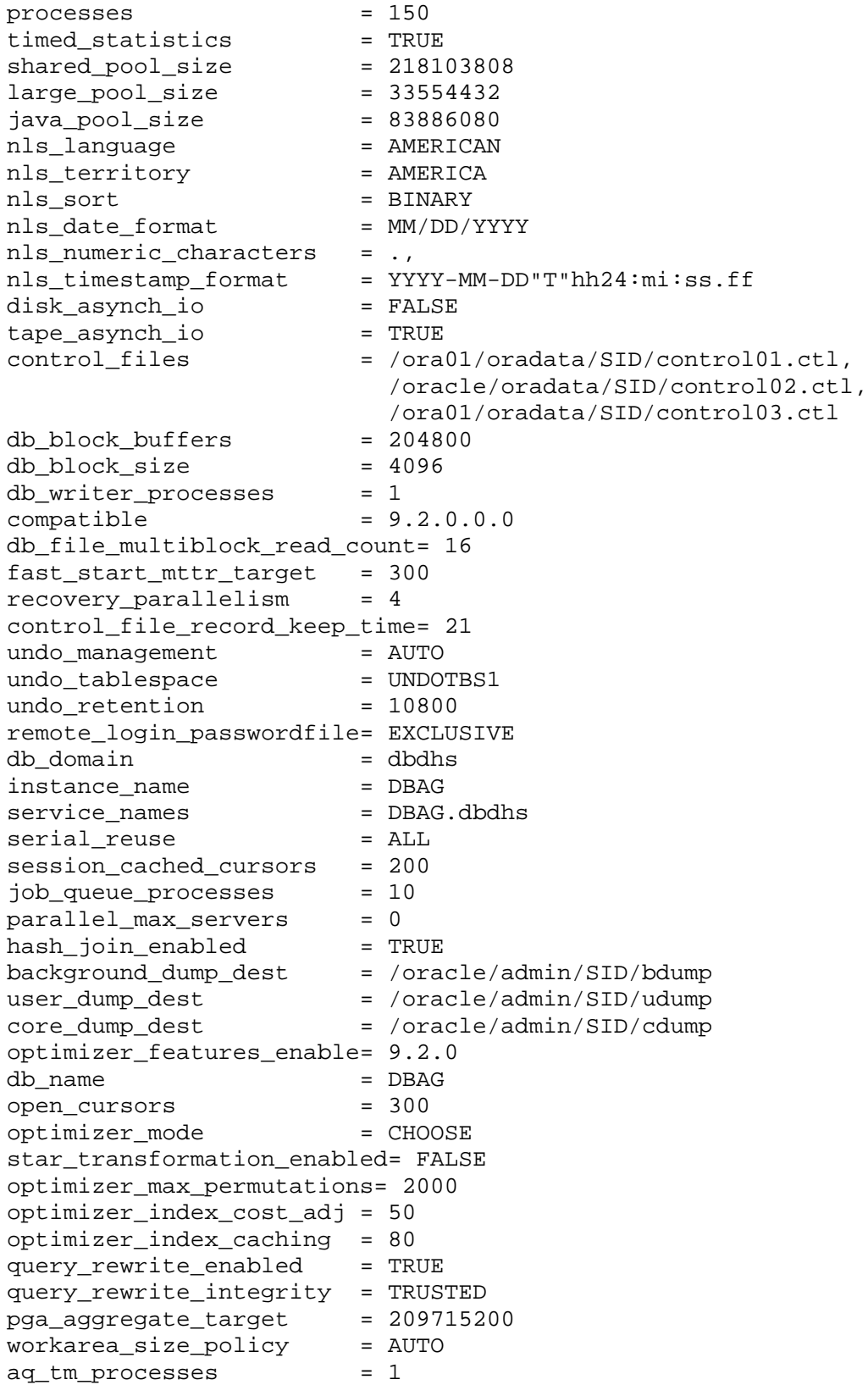




\section{References}

Note: 34592.1, WAITEVENT: "log file sync" Reference Note, 11-NOV-2002, Oracle Corporation Hotsos Profiler, Hotsos Enterprises, Ltd., www.hotsos.com

Optimizing Oracle Performance, September 2003, Cary Millsap with Jeff Holt, O'Reilly

Oracle File System Integration and Performance, January 2001, Richard McDougall, Sriram Gummuluru, Sun Microsystems

Evaluation of VERITAS File System to Enhance Oracle RDBMS Performance, Version 1.0, August 23, 1999, Roby Sherman

File System Performance White Paper, Part 1, 1996, VERITAS Software Corporation

File System Performance White Paper, Part 2, 1996, VERITAS Software Corporation

File System Performance White Paper, Part 3, 1996, VERITAS Software Corporation

Tips and Techniques: Install and Configure Oracle9i on Red Hat Linux Advanced Server, An Oracle White Paper, August 2002, Oracle Corporation

Tuning Oracle Database Server and Linux, Part 1, Bert Scalzo, Quest Software

Tuning an Oracle8i Database Running Linux, Part 2, Bert Scalzo, Quest Software

LINUX Maximus Part 2: The RAW Facts on File Systems, Bert Scalzo, Quest Software 